\title{
Impact of pantoprazole on absorption and disposition of hydroxychloroquine, a drug used in Corona Virus Disease-19 (Covid-19): A structured summary of a study protocol for a randomised controlled trial
}

Felicitas Stoll ${ }^{\dagger}$, Antje Blank ${ }^{* \dagger}$ (1) Gerd Mikus, David Czock, Kathrin I. Foerster, Simon Hermann, Katja Häußler, Amin Muhareb, Simone Hummler, Johanna Weiss, Jürgen Burhenne and Walter E. Haefeli

\footnotetext{
Abstract

Objectives: Primary objective: Evaluation of the effect of the proton pump inhibitor (PPI) pantoprazole on the absorption of hydroxychloroquine (HCQ).

Secondary objectives:

- Evaluation of the relationship between HCQ concentrations in whole blood, plasma and intracellular concentrations in target cells - peripheral blood mononuclear cells (PBMCs).

- Evaluation of HCQ as a potential perpetrator in drug-drug interactions at the level of cytochrome P450 (CYP) 3A4 and CYP2D6 (major drug metabolizing enzymes).

Trial design: Single centre, open-label, parallel group, two-arm, phase I drug-drug interaction trial.

Participants: Healthy volunteers (18-60 years old) are treated in the Clinical Pharmacological Trial Center of Heidelberg University Hospital, Germany.

Intervention and comparator: • Participants are randomized in a group to either receive a nine-day course of pantoprazole, or to a control group without pantoprazole. All participants receive a single dose of HCQ $400 \mathrm{mg}$.

- Additionally, CYP3A4 and CYP2D6 phenotyping with microdosed probe drugs is performed using midazolam and yohimbine as enzyme activity markers, respectively.

Main outcomes: Primary endpoint:

Area under the curve $(A \cup C)_{0-72} \mathrm{~h}$ and maximum concentration $\left(\mathrm{C}_{\max }\right)$ of a single oral dose of $400 \mathrm{mg} \mathrm{HCQ}$ with and without pantoprazole (changes in these two values describe relevant aspects of exposure to HCQ with and without administration of pantoprazole).

Secondary endpoints:

- $\mathrm{AUC}_{2-4} \mathrm{~h}, \mathrm{AUC}_{0-6} \mathrm{~h}$ and $\mathrm{C}_{\max }$ of midazolam and yohimbine.

(Continued on next page)
}

\footnotetext{
* Correspondence: antje.blank@med.uni-heidelberg.de

${ }^{\dagger}$ Felicitas Stoll and Antje Blank are joint first authors.

Heidelberg University Hospital, Heidelberg, Baden-Württemberg, Germany
}

C The Author(s). 2020 Open Access This article is licensed under a Creative Commons Attribution 4.0 International License, which permits use, sharing, adaptation, distribution and reproduction in any medium or format, as long as you give appropriate credit to the original author(s) and the source, provide a link to the Creative Commons licence, and indicate if changes were made. The images or other third party material in this article are included in the article's Creative Commons licence, unless indicated otherwise in a credit line to the material. If material is not included in the article's Creative Commons licence and your intended use is not permitted by statutory regulation or exceeds the permitted use, you will need to obtain permission directly from the copyright holder. To view a copy of this licence, visit http://creativecommons.org/licenses/by/4.0/. The Creative Commons Public Domain Dedication waiver (http://creativecommons.org/publicdomain/zero/1.0/) applies to the data made available in this article, unless otherwise stated in a credit line to the data. 
(Continued from previous page)

- Correlation of HCQ concentrations in whole blood with concentrations in plasma and peripheral blood mononuclear cells (PBMC).

Randomisation: Participants are assigned to treatment groups by using a randomisation list $(1: 1$, block size $=4)$ and consecutive enrolment.

Blinding (masking): The trial is an open-label trial, participants and investigators are not blinded.

Numbers to be randomised (sample size): A total number of 24 participants (12 per group) are planned to be randomised.

Trial Status: Protocol version 2.1 dated 24/04/2020, first patient first visit. April 30th, 2020, recruitment ongoing, anticipated end of study June 30th, 2020.

Trial registration: EudraCT Number: 2020-001470-30, registered on 31 March 2020 German Clinical trials register number / International Clinical Trials Registry Platform: DRKS00021573, registered on 27 April 2020

Full protocol: The full trial protocol is attached as an additional file, accessible from the Trials website (Additional file 1). In the interest in expediting dissemination of this material, the familiar formatting has been eliminated; this Letter serves as a summary of the key elements of the full trial protocol. The trial protocol has been reported in accordance with the Standard Protocol Items: Recommendations for Clinical Interventional Trials (SPIRIT) guidelines (Additional file 2).

Keywords: COVID-19, Randomised controlled trial, Trial protocol, Hydroxychloroquine, Pantoprazole, Absorption, Drug-drug interaction, Microdosing, Midazolam, Yohimbine

\section{Acknowledgements}

We express our sincere gratitude to the study nurses of the Clinical Pharmacological Trial Center, Katharina Hilse, Florian Michel, Sophie Sherman, Marlies Stützle-Schnetz, and Brigitte Tayrouz for their excellent technical support during trial implementation and conduct. We also thank the clinical monitors Viktoria Jungreithmayr and Robert Möcker for their thorough supervision throughout the trial.

\section{Authors' contributions}

FS and $A B$ : writing of trial protocol and manuscript, deputy investigators, conduct of the trial. WEH: conception of the trial, writing of trial protocol, principal investigator, conduct of the trial. GM: conception of the trial, writing of trial protocol, biostatistician. DC: conception of the trial, writing of trial protocol. $\mathrm{AMu}, \mathrm{KSH}, \mathrm{SH}, \mathrm{SHu}$ : sub- investigators conducting the trial. KIF, JB, JW: laboratory methods and evaluation. The author(s) read and approved the final manuscript.

\section{Authors' information}

WEH, AB, FS, GM and DC are experts in the field of clinical pharmacology and focus on optimization of drug therapy and safety of drug treatments.

\section{Funding}

The study is funded by the Department of Clinical Pharmacology and Pharmacoepidemiology of Heidelberg University Hospital, Germany. The legal sponsor of the trial is the Ruprecht-Karls-University Heidelberg, Medical Faculty, represented in law by Heidelberg University Hospital, Germany, and its commercial director Katrin Erk. The trial is investigator-initiated, therefore, the main investigator has obtained all rights and duties from the legal sponsor except contract management. The legal sponsor itself does not have a role in collection, management, analysis, and interpretation of data; writing of the report; and the decision to submit the report for publication.

\section{Availability of data and materials}

Data will be available from the author on reasonable request.

\section{Ethics approval and consent to participate}

The study was approved by the responsible Ethics Committee of the Medical Faculty of Heidelberg University on 17/04/2020 (AFmo-265/2020) and by the competent authority (Federal Institute for Drugs and Medical Devices, BfArM, Bonn, Germany) on 23/04/2020. All volunteers are fully informed about the trial, are free to participate, and are asked to give their written consent prior to any trial-related procedures. We certify that this trial has received ethical approval from the appropriate Ethics Committee as described above.

Consent for publication

Not applicable.

\section{Competing interests}

The authors declare that they have no competing interests.

Received: 29 May 2020 Accepted: 31 May 2020

Published online: 29 June 2020

\section{Supplementary information}

Supplementary information accompanies this paper at https://doi.org/10 1186/s13063-020-04476-y.

Additional file 1. Full Study Protocol.

Additional file 2. SPIRIT 2013 Checklist: Recommended items to address in a clinical trial protocol and related documents.

Received: 29 May 2020 Accepted: 31 May 2020

Published online: 29 June 2020

\section{Publisher's Note}

Springer Nature remains neutral with regard to jurisdictional claims in published maps and institutional affiliations. 